\title{
POST-NATAL CHANGES IN THE INTRA-ABDOMINAL UMBILICAL VEIN
}

\author{
BY \\ H. BUTLER \\ From the Department of Anatomy, St. Bartholomew's Hospital Medical College, London
}

(RECEIVED FOR PUBLICATION APRIL 12, 1954)

Recent investigations of the birth changes in the foetal circulation indicate that certain channels, namely, the ductus arteriosus, ductus venosus and extra-abdominal umbilical vessels, are quickly closed by the contraction of their muscular walls (Barclay, Franklin and Prichard, 1944). This immediate functional closure is followed by an anatomical obliteration which takes place during the first few weeks or months of life (Scammon and Norris, 1918; Kennedy and Clark, 1941). Little appears to be known regarding the functional response to birth changes of the intra-abdominal umbilical vein, but Barclay et al. (1944) note the following facts.

First, that there is a marked difference between the structure of the intra- and extra-abdominal parts of the umbilical vein: the former is thin-walled with only a small amount of smooth muscle, whereas the latter has a very thick muscular wall. Second, that the intra-abdominal umbilical vein is much reduced in calibre and contains little or no blood when examined some time after rupture of the cord.

Sidbury (1923) first used the umbilical vein as a transfusion route (in infants suffering from icterus gravis neonatorum) by inserting a needle into the extra-abdominal umbilical vein just beyond the ligature on the cord. He found no clot in the vein and attributed this to the delay in clotting which is a characteristic of this condition. Diamond (1947) elaborated this method by severing the umbilical cord, some half-inch from the abdominal wall, and inserting a polythene catheter of $1 \mathrm{~mm}$. internal diameter directly into the intra-abdominal umbilical vein. This technique is now the standard one for performing exchange transfusions in icterus gravis neonatorum and may be employed for as long as six days after birth (Diamond, 1954). When the cord is severed the vein occasionally bleeds violently, but 'if, as is usual, the vein does not bleed at all, it can be identified as a large patulous vessel which contrasts with the two smaller, tightly contracted arteries' (Mollison, Mourant and Race, 1948). The catheter meets no resistance until it impinges upon the far wall of the left branch of the portal vein and it may even be introduced into the ductus venosus and so into the inferior vena cava.

Much information is available regarding the mode of anatomical obliteration of the intra-abdominal umbilical vein and the formation of the ligamentum teres hepatis (Robin, 1860; Wertheimer, 1886; Baumgarten, 1891; Butler, 1951). The account by Robin (1860) does not depict the relevant histological changes, so that interpretation of the obscure text becomes well-nigh impossible, and it is probable that sepsis was present in much of his young material. Wertheimer (1886) describes the formation of a central core of fibrous tissue completely filling the lumen of the vein. The obliterated lumen becomes surrounded by a musculo-elastic ring, while capillary formation in the central core of the fibrous tissue gives the spurious appearance of a tiny remnant of the original lumen of the vein. It is important to note that the youngest specimen described was from an infant aged 5 months. Baumgarten (1891) states that the lumen of the umbilical vein is not completely obliterated, and that a much reduced remnant, the Rest-Kanal, is to be found in most individuals. This opens into the left branch of the portal vein and extends for a varying distance along the ligamentum teres. Segall (1923) demonstrated this small vessel in $40 \%$ of injected adult human livers. In my opinion (Butler, 1951; 1952) the latter view is the correct one and is confirmed by the following observations, made on very young specimens. These observations also show why it is possible to use the intra-abdominal vein as a transfusion route in the early days of life.

\section{MATERIAL AND METHODS}

Thirty-one specimens of the intra-abdominal umbilical vein and ligamentum teres were examined; they ranged in age from birth to 77 years, but only 
the 20 specimens between birth and 8 years are here considered in detail. Transverse sections were cut, in all specimens, at a point approximately midway between the umbilical ring and the left branch of the portal vein. In addition to routine haematoxylin and eosin staining, all sections were stained by the Weigert-van Gieson method for smooth muscle and elastic tissue.

The dimensions of certain sections were measured in the following manner. An image of the section, magnified 25 times (linear), was projected on to squared graph paper and its outline carefully delineated. The following areas were ascertained by counting the number of enclosed squares and converting the results into square millimetres:

A . . Either (1) the cross-sectional area of the wall of the intra-abdominal umbilical vein, or (2) the cross-sectional area of the ligamentum teres

L . . The cross-sectional area of the lumen of the intra-abdominal umbilical vein

1 ... The cross-sectional area of the residual lumen of the intra-abdominal umbilical vein found in the centre of the ligamentum teres

a ... The area enclosed by the musculo-elastic ring, i.e., the area of the central core of new fibrous tissue

Assuming the vein to be of circular cross-section during life, an estimate of its internal diameter (D) was made. The thickness of the vein wall $(T)$ was measured directly with an eyepiece micrometer. Owing to the manner in which the specimens had been removed after death, it was not possible to measure the length of the vein or the ligament.

\section{OBSERVATIONS}

Intra-abdominal Umbilical Vein between Birth and 13 Days

Dimensions. Table 1 lists the dimensions of nine of the 14 specimens included in this age group.

TABLE 1

DIMENSIONS OF SPECIMENS AGED 0 TO 13 DAYS

\begin{tabular}{|c|c|c|c|c|c|c|}
\hline \multicolumn{2}{|c|}{ Number } & Age & $\underset{\text { (sq. } \mathrm{mm} \text {.) }}{\mathrm{A}}$ & $\underset{\text { (sq. } \mathrm{mm} \text {.) }}{\mathbf{L}}$ & $\underset{(\mathrm{mm} .)}{\mathbf{D}}$ & $\underset{(\mathrm{mm} .)}{\mathbf{T}}$ \\
\hline Type A & $\begin{array}{l}1 \\
5 \\
6\end{array}$ & $\begin{array}{l}\text { Full term } \\
45 \text { minutes } \\
6 \text { hours }\end{array}$ & $\begin{array}{l}6 \cdot 75 \\
7.72 \\
3 \cdot 22\end{array}$ & $\begin{array}{l}4 \cdot 50 \\
3 \cdot 77 \\
3 \cdot 92\end{array}$ & $\begin{array}{l}2 \cdot 5 \\
2 \cdot 2 \\
2 \cdot 3\end{array}$ & $\begin{array}{l}0.515 \\
0.700 \\
0.220\end{array}$ \\
\hline Type B & $\begin{array}{r}3 \\
7 \\
8 \\
9 \\
10 \\
11\end{array}$ & $\begin{array}{l}\text { Full term } \\
10 \text { hours } \\
19 \text { hours } \\
28 \frac{3}{4} \text { hours } \\
48 \text { hours } \\
48 \text { hours }\end{array}$ & $\begin{array}{l}8 \cdot 65 \\
5 \cdot 77 \\
7 \cdot 12 \\
5 \cdot 32 \\
4 \cdot 71 \\
4 \cdot 73\end{array}$ & $\begin{array}{l}1.41 \\
0.61 \\
0.65 \\
0.88 \\
1.34 \\
0.60\end{array}$ & $\begin{array}{l}1.4 \\
0.9 \\
0.9 \\
1.1 \\
1.3 \\
0.9\end{array}$ & $\begin{array}{l}0.825 \\
0.880 \\
0.810 \\
1.045 \\
0.750 \\
0.750\end{array}$ \\
\hline
\end{tabular}

The remaining five specimens, having been opened, could not be measured accurately, but their general appearances indicate that they fall into one or other of the two types. All specimens show a widely patent lumen and none shows any evidence of ante-mortem thrombosis. Type A specimens are characterized by a thin wall and a large lumen; type B specimens have a thicker wall and a proportionately smaller lumen. These proportions obtain whether the vessels be circular or oval in crosssection (Fig. 1). With the exception of specimen 3, the thin-walled veins are younger than the thickwalled.

Comparison of the average dimensions of the two types clearly indicates that considerable changes in size of lumen and wall-thickness are accompanied by a negligible change in the cross-sectional area of the vessel wall (Table 2). This indicates that the

TABLE 2

AVERAGE DIMENSIONS

\begin{tabular}{|c|c|c|c|c|c|}
\hline \multirow[b]{2}{*}{$\begin{array}{l}\mathbf{A} \\
\mathbf{L} \\
\mathbf{D} \\
\mathbf{T}\end{array}$} & \multicolumn{2}{|c|}{ Type A } & \multicolumn{2}{|c|}{ Type B } & \multirow{2}{*}{$\begin{array}{c}\text { Difference (\%) } \\
4 \text { increase } \\
78 \text { increase } \\
57 \text { decrease } \\
63 \text { increase }\end{array}$} \\
\hline & $\begin{array}{l}5 \cdot 80 \\
4 \cdot 16 \\
2 \cdot 33 \\
0 \cdot 515\end{array}$ & $\begin{array}{l}\text { sq.mm. } \\
\text { sq.mm. } \\
\text { mm. } \\
\text { mm. }\end{array}$ & $\begin{array}{l}6 \cdot 05 \\
0.91 \\
1 \cdot 10 \\
0.842\end{array}$ & $\begin{array}{l}\text { sq.mm. } \\
\text { sq.mm. } \\
\text { mm. } \\
\text { mm. }\end{array}$ & \\
\hline
\end{tabular}

vein has undergone a partial contraction, reducing the average diameter of the lumen from $2 \cdot 33$ to $1.1 \mathrm{~mm}$. If allowance be made for shrinkage due to fixation and dehydration it is clear that the lumen of the partially contracted intra-abdominal umbilical vein is of the same order of size as the polythene catheter used for exchange transfusion. The older specimens, aged $3 \frac{1}{2}$ to 13 days, were not measurable accurately, but the estimated dimensions of their lumina and walls are in keeping with those found for type B. No accurate indication of the time of occurrence of the partial contraction, in relation to the onset of respiration, can be gathered from the material available. All specimens older than 10 hours are in a state of partial contraction.

Histological Appearances. The histological appearances of the two types of vein completely accord with the view that the dimensional changes are due to the contraction of the intramural smooth muscle.

Type $A$. The wall of the intra-abdominal part of the umbilical vein does not exhibit the classic picture of tunica intima, tunica media and tunica adventitia (Fig. 2, 1; Fig. 3, 1) and is best described as follows. Immediately beneath the endothelium is a narrow musculo-elastic zone containing a layer of longitudinal smooth muscle, one to two cells deep. Interspersed among the smooth muscle fibres is a longitudinal network of elastic fibres, forming a thin (occasionally incomplete) internal elastic layer. In section this layer 

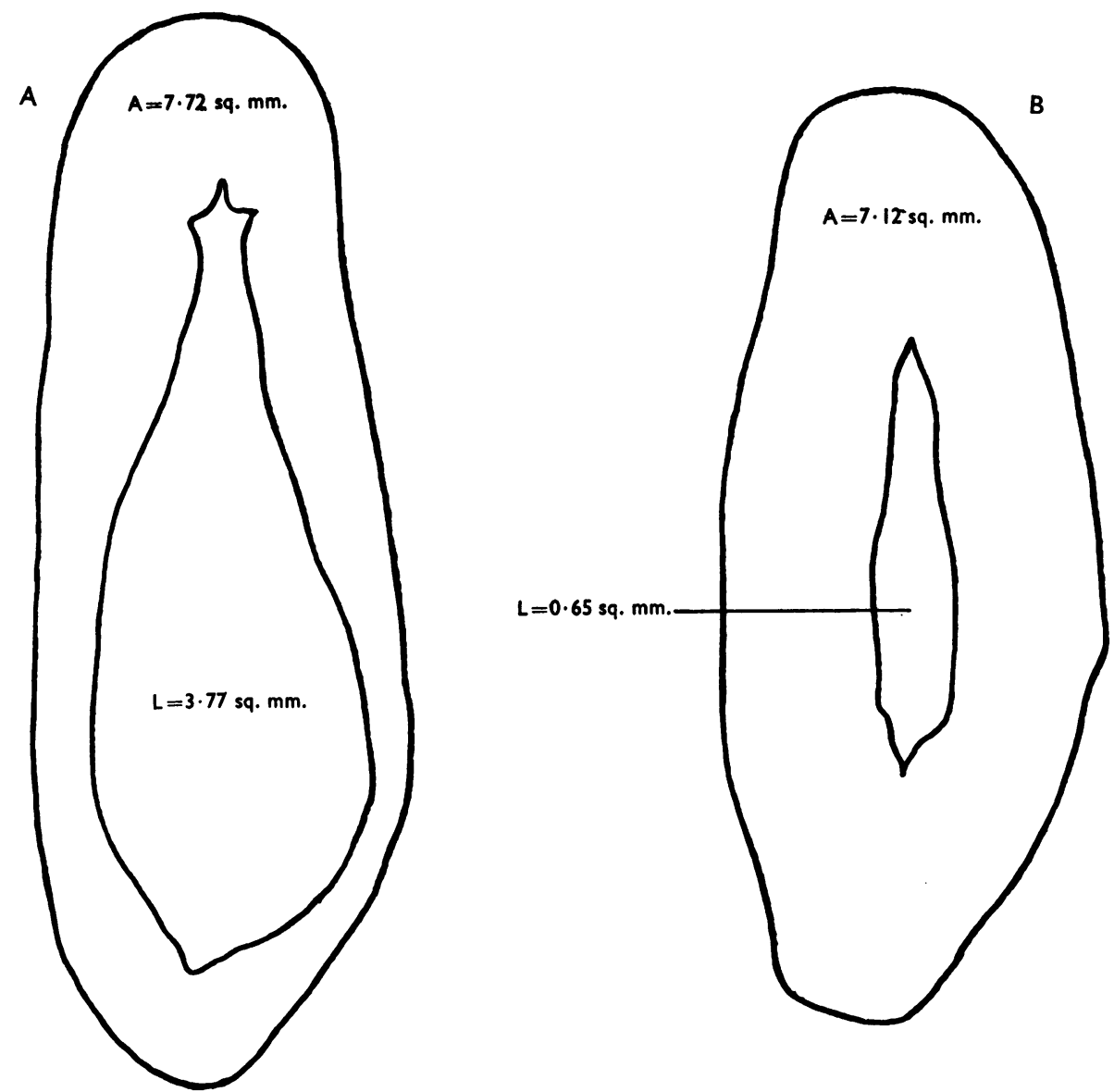

$\mathrm{T}$ (average) $=700 \mu$

$D$ (calculated) $=2 \cdot 2 \mathrm{~mm}$. Specimen No. 5 45 mins.

$\mathrm{T}$ (average $)=810 \mu$

$D$ (calculated $)=0.9 \mathrm{~mm}$.

Specimen No. 8

19 hours
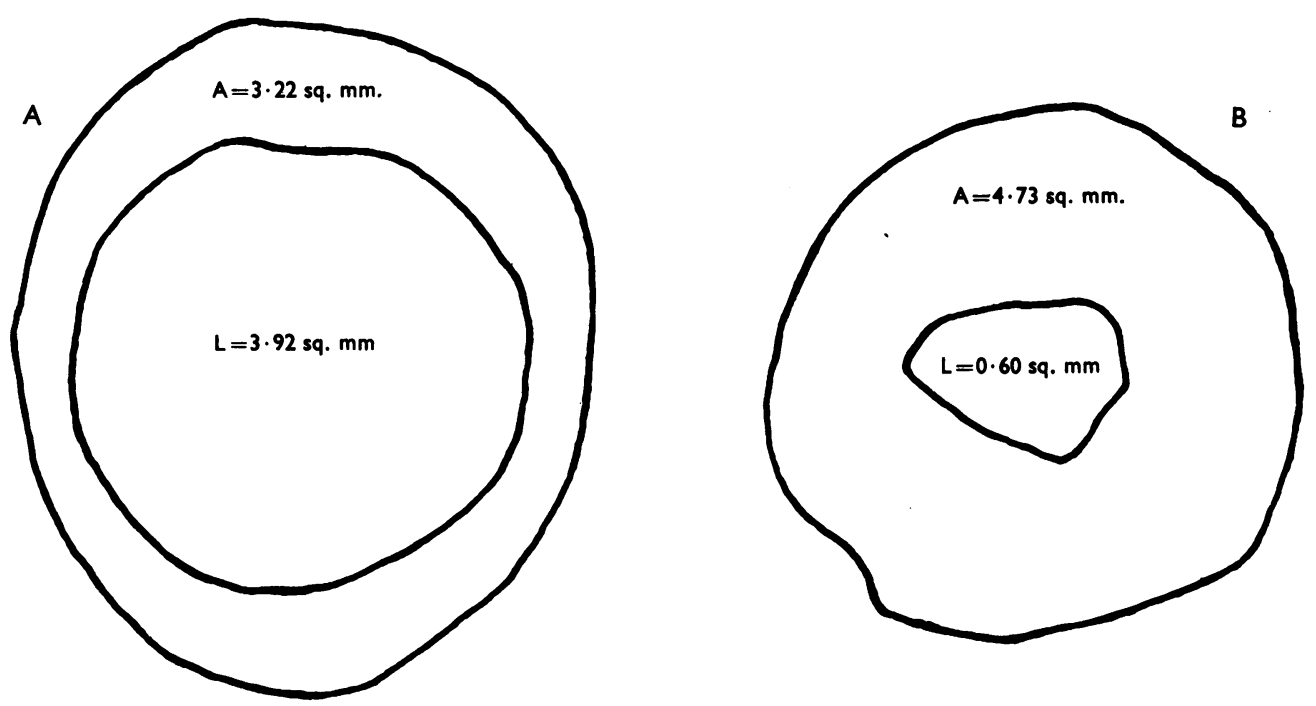

$T$ (average) $=220 \mu$

$D$ (calculated $=2 \cdot 3 \mathrm{~mm}$.

Specimen No. 6 6 hours

$T$ (average) $=750 \mu$

$D$ (calculated $=0.9 \mathrm{~mm}$.

Specimen No. II 48 hours

FIG. 1.-Tracings of sections of the intra-abdominal umbilical vein in the relaxed (type A) and contracted (type B) states. Examples of the circular and ovoid types are shown. All $\times 25$.

Photomicrographs of specimens 6 and 11 are shown in Fig. 2 (1 and 2) and Fig. 3 (1 and 2). 


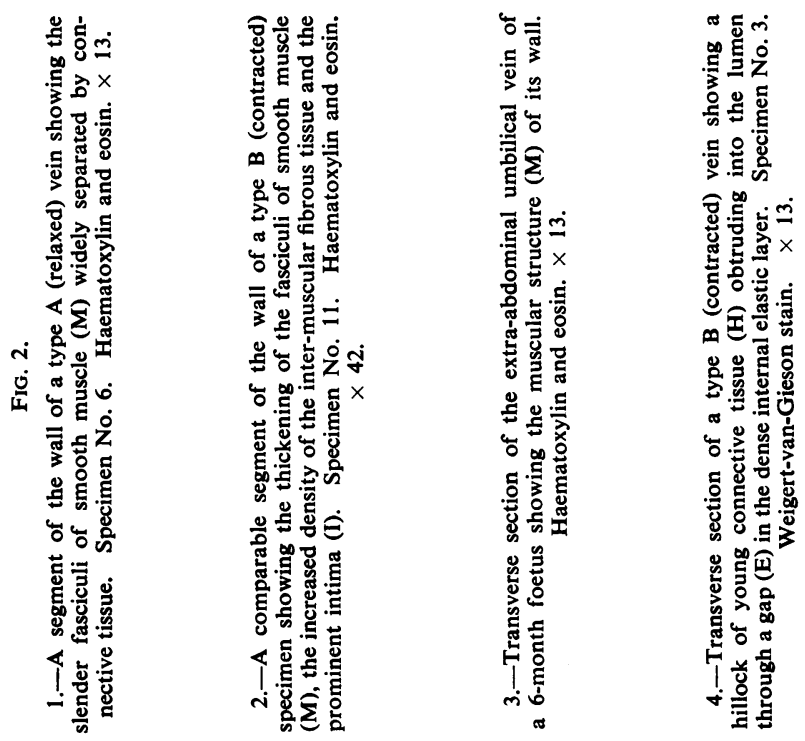
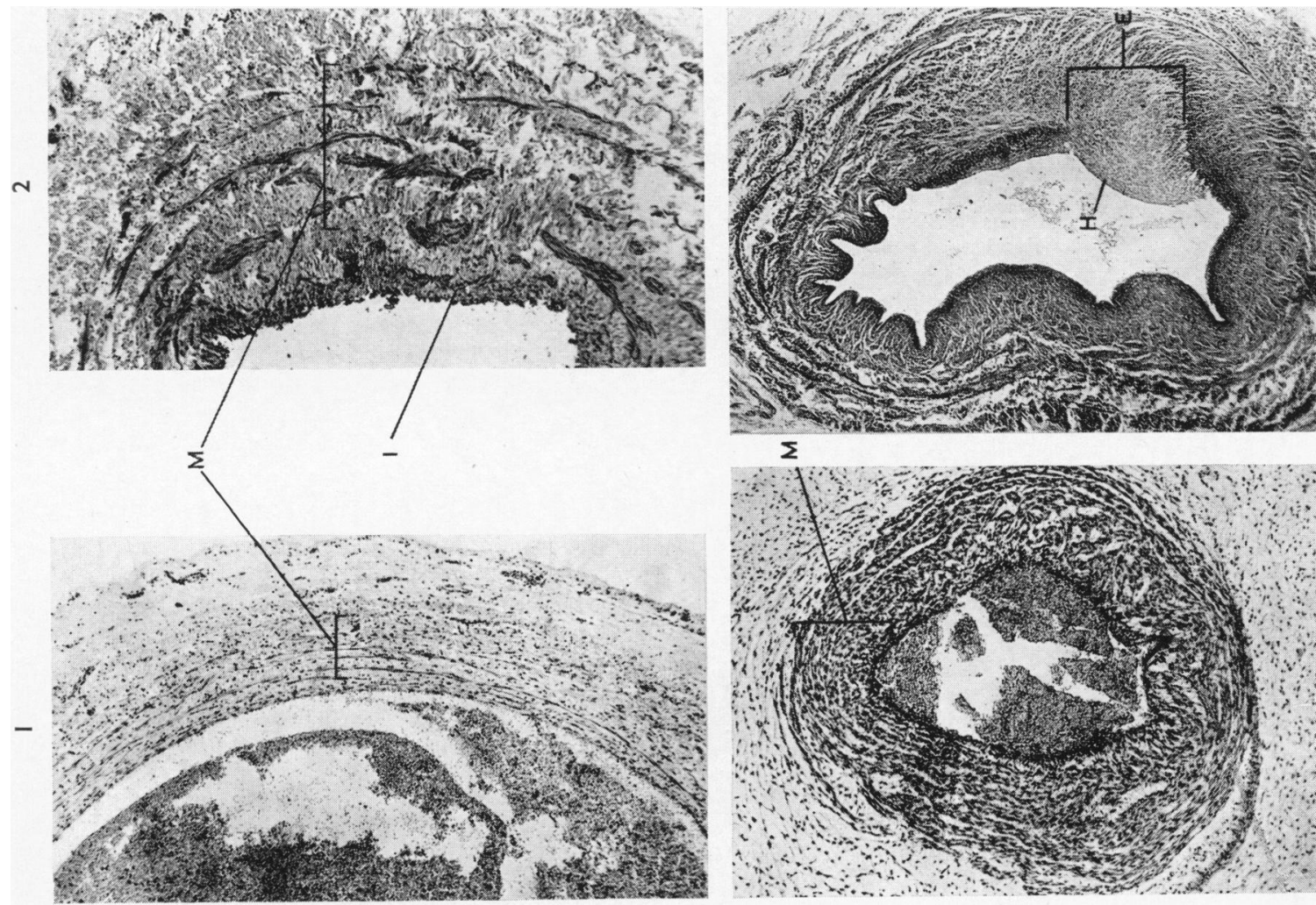

$\Sigma$

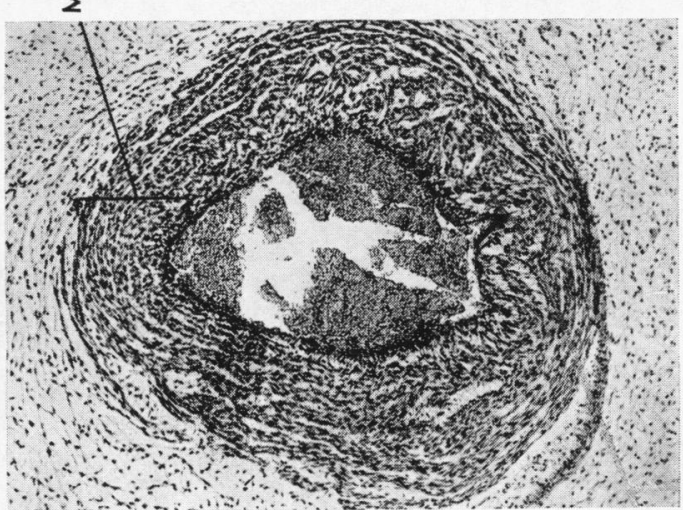


appears as a row of black dots immediately adjacent to the endothelium. The main bulk of the vessel wall consists of white fibrous tissue containing fasciculi of smooth muscle fibres (Fig. 2, 1), arranged in open spirals widely separated by white fibrous tissue. Intimately associated with the muscle fasiculi are long elastic fibres disposed parallel to the long axis of the muscle fibres. This main part of the vein wall forms a fibro-muscular media which, on its inner aspect, merges with the musculo-elastic zone. Externally the tunica media presents a clear-cut margin (Fig. 3, 1) surrounded by fine, white connective tissue-the extra-peritoneal tissue of the falciform ligament. This contains numerous blood vessels and nerves and takes the place of a conventional tunica adventitia.

Type B. General features are similar to those of type A, but certain structures are rendered more prominent by the state of partial contraction. The spiral muscle fasciculi of the media are more obvious: they appear to run a more circular course and to be set closer together (Fig. 2, 2; Fig. 3, 2). Individual muscle fibres are bulkier and shorter, and their appearance is unchanged at the thirteenth day after birth. The muscle content of the intra-abdominal umbilical vein is considerably less than that seen in the extra-abdominal umbilical vein of a six-months foetus (Fig. 2, 3)

The musculo-elastic zone, particularly its elastic fibres, is more obvious since its components are now crowded within a much smaller perimeter. The elastic fibres appear as a zone of black dots, five or six deep, immediately next to the endothelium (Fig. 2, 4; Fig. 3, 2). This prominent musculo-elastic zone remains visible for almost the whole of life as an easily identifiable landmark, indicating the position of the inner surface of the partially contracted intra-abdominal umbilical vein. Further evidence of contraction is seen in the crenation of the inner margin of the vein and the close apposition of the nuclei of the endothelial cells. Where a gap occurs in the musculoelastic zone a small hillock of young connective tissue projects into the vessel lumen (Fig. 2, 4). The bases of these connective tissue hillocks extend outwards into the fibro-muscular media. The hillocks are the result of sub-intimal proliferation which begins before birth and the new tissue is, as it were, extruded into the lumen through the gaps in the musculo-elastic zone when the vessel contracts. Their appearance marks the beginning of the lengthy process of anatomical obliteration of the vessel lumen.

The intra-abdominal umbilical vein receives a number of small tributaries, namely its own vena venarum and the veins of Burow. The latter connect the epigastric venous plexus with the portal venous system via the intra-abdominal umbilical vein, and are relatively much larger in the foetus and neonatus than in the adult. They contain valves which direct the blood-flow towards the portal vein. It is therefore most probable that the blood found in the partially contracted intra-abdominal umbilical vein is an inflow from these tributaries and not a backflow from the portal vein.

\section{Formation of the Ligamentum Teres from 1 Month Onwards}

The various stages in the formation of the ligamentum teres are seen in specimens aged from 1 month to 8 years. Three main and simultaneous processes seem to be involved in the further reduction of the lumen of the umbilical vein. These are as follows :-

Side-to-side Flattening of the Vein. This process, already present in the very young specimens, is most marked at 1 month (Fig. 2, 3). In this specimen the lumen is reduced to a cleft about $3.0 \mathrm{~mm}$. long with its long axis in the sagittal plane. The sides of the cleft are in contact for some twothirds of its length and in some places the apposed walls are united by newly formed white fibrous tissue. The maximum diameter of the lumen is seen to be about 80 to $100 \mu$.

Formation of New Connective Tissue inside the Lumen. This is achieved by the continued growth of the hillocks protruding through the gaps in the musculo-elastic zone. This new tissue spreads irregularly around the lumen of the vein, always disposed between the endothelium and the musculoelastic zone and thus forming the central core of the fibrous tissue first described by Wertheimer (1886). The patent lumen of the vein becomes greatly reduced but never completely obliterated (Fig. 3, 3, 4, 5 and 6). At first the newly formed fibrous tissue consists solely of the white fibres but, from 8 years on, a few small and irregularly disposed elastic fibres are found. The bases of the hillocks, embedded in the fibro-muscular media, lay down much coarser collagen fibres and these eventually become indistinguishable from those which form the bulk of the ligamentum teres.

Composition of the Tunica Media. From 1 month onwards the smooth-muscle spiral fasciculi become distorted and disrupted by the increasing collagenous tissue composed of coarse collagen fibres. Interspersed among the collagen fibres are moderately 


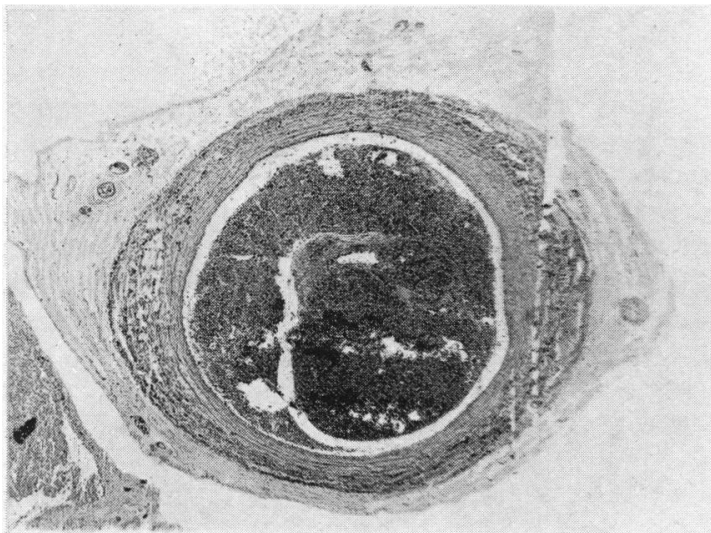

।

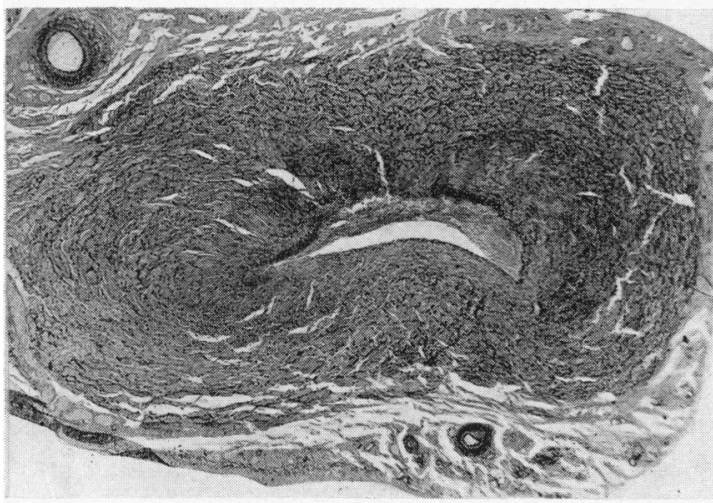

3

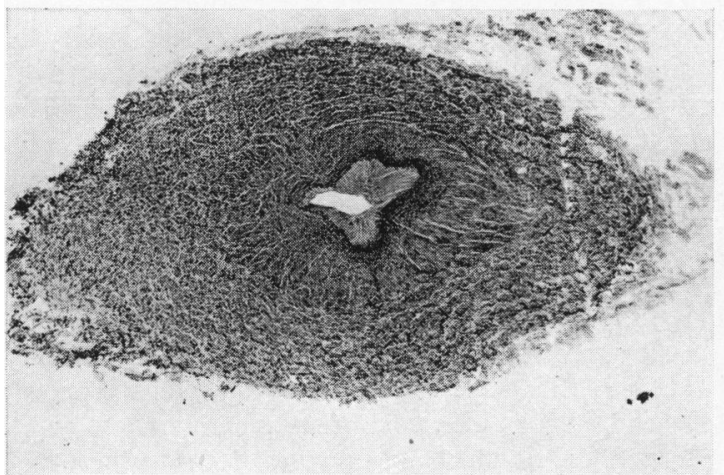

5

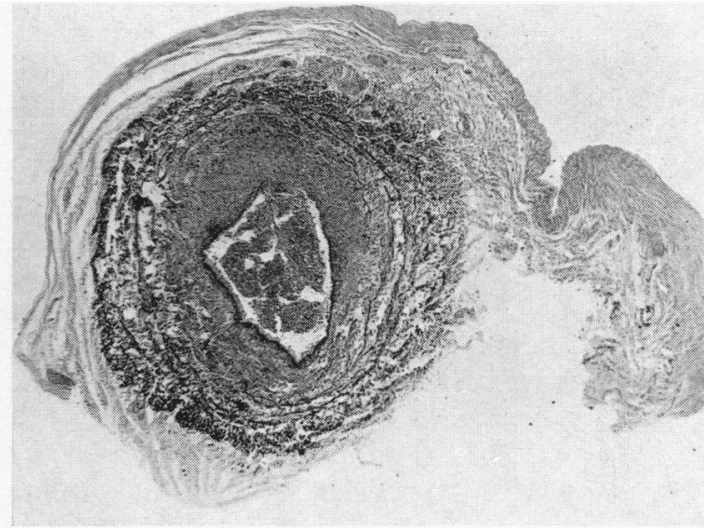

2

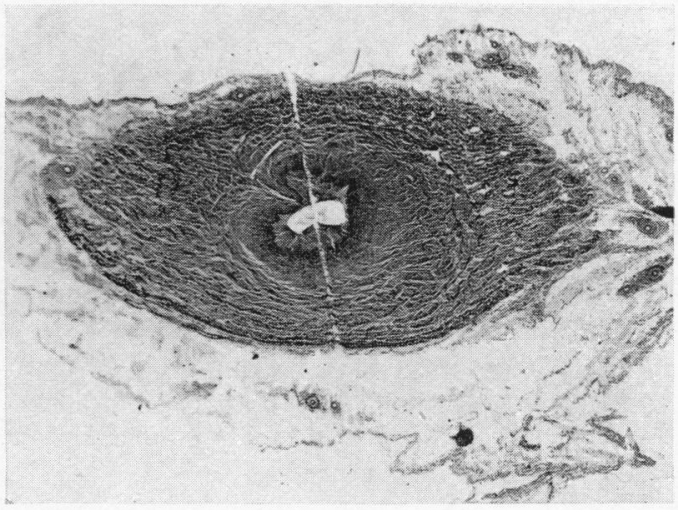

4



6

Fig. 3.-Cross sections of the intra-abdominal umbilical vein and ligamentum teres from birth to 8 years. Weigert-van Gieson. All $\times 13$.

1.-Type A (relaxed) vein, specimen No. 6, 45 minutes old, showing the slender, widely separated muscle fasciculi and the tenuous internal elastic layer.

2.-Type B (contracted) vein, specimen No. 11, 48 hours old, showing the thickening of the muscle fasciculi and the increased density and width of the internal elastic layer. The latter shows a gap at the upper aspect of the lumen associated with a small hillock of new tissue (cf. Fig. 2, 4).

3.-Ligamentum teres, specimen No. 16, 28 days old, showing side-to-side flattening of the lumen and the increased amount of new connective tissue. This latter has clearly spread inside the internal elastic layer.
4.-Ligamentum teres, specimen No. 15, 3 months old, the final zonal pattern is now clearly established (see Fig. 4) and, as a result of shrinkage, the musculo-elastic zone is crenated and covers a much reduced perimeter.

5.-Ligamentum teres, specimen No. 19,13 months old, the histological pattern is exactly as in the previous specimen.

6.-Ligamentum teres, specimen No. 20,8 years old, showing increased reduction in the residual lumen and further contraction of the musculo-elastic zone. The main fibrous zone of the ligament now contains more elastic tissue and fat deposits at its periphery. 
large longitudinal elastic fibres whose numbers increase with age. Coincident with these changes the ligament becomes increasingly vascular, particularly at its periphery and, by 8 years, fat cells are seen among the marginal collagen fibres (Fig. 3, 6). As the white fibrous tissue of the ligamentum teres increases in bulk so the area enclosed by the musculo-elastic ring decreases.

Swelling of the spiral muscle fibres and alterations in their staining properties indicate the onset of degenerative changes. In the older specimens these muscle fibres become increasingly difficult to find and can often be located only by the presence of the persistent spiral elastic fibres surrounding each muscle fasciculus. Between 3 and 6 months numerous mononuclear phagocytes are to be found around the degenerating muscle fibres. A few poorly staining and obviously degenerate muscle fibres are seen in the 8-year-old specimen but after this age they are no longer detectable. From 20 years onwards small, widely-spaced fasciculi of longitudinal smooth muscle appear amongst the collagen fibres and have all the appearance of a new formation.

Measurements of the cross-sectional area of the intra-abdominal umbilical vein and of the ligamentum teres indicate that growth of the ligament begins during the first year of life and attains its maximum by about 18 years, thereafter remaining constant (Table 3).

TABLE 3

GROWTH OF THE LIGAMENTUM TERES

\begin{tabular}{ccc|c}
\hline Age & & & A (sq.mm., average) \\
\hline Birth, type A &.. & $\ldots$ & $5 \cdot 80$ \\
Birth, type B & $\ldots$ & $\ldots$ & 6.05 \\
1 to 6 months & $\ldots$ & $\ldots$ & 5.89 \\
1 to 8 years & $\ldots$ & $\ldots$ & 13.05 \\
13 to 18 years & $\ldots$ & $\ldots$ & 12.65 \\
31 to 63 years &. &. & \\
\hline
\end{tabular}

The musculo-elastic zone remains clearly visible into the sixth decade by reason of the persistence of its elastic fibres. From 3 months onwards these elastic fibres become packed into an ever-decreasing perimeter until, at about 20 years, they become mutually separated by the formation of a ring of longitudinal smooth muscle fibres. It is probable, but not certain, that the original muscle fibres of this zone disappear during the first eight years of life and that the ring of muscle seen from 20 years onwards is an entirely new formation. As the elastic fibres become more closely packed together they form a crenated ring and enclose a reduced area. This area (L) is, of course, the equivalent of the area of the lumen of the intra-abdominal umbilical vein and it reaches its maximum reduction by about 8 years of age (Table 4). In its centre, and separated from the musculo-elastic ring by the fibrous tissue core, is the patent remnant of the intra-abdominal vein (l). This lumen, also, undergoes progressive reduction in size and by 8 years it has a cross-sectional area of 0.005 sq.mm. This lumen is present in all the specimens examined, up to the sixth decade, and its area varies between 0.005 and 0.22 sq.mm. It is always maximal at the hepatic end of the ligamentum teres and shows a tendency to increase slightly with age. In portal venous obstruction it may become greatly enlarged, attaining a diameter even greater than that of the original intra-abdominal umbilical vein (Butler, 1952).

\section{TABLE 4}

\begin{tabular}{l|c|c|c}
\hline & Age & $\begin{array}{c}\text { L } \\
\text { (sq.mm.) }\end{array}$ & $\begin{array}{c}1 \\
\text { (sq.mm.) }\end{array}$ \\
\hline Type A (average) & Birth & $4 \cdot 16$ & $4 \cdot 16$ \\
Type B (average) & Birth & 0.91 & 0.91 \\
Specimen No. 16 & 1 month & 0.47 & 0.07 \\
Specimen No. 15 & 3 months & 0.25 & 0.06 \\
Specimen No. 19 & 13 months & 0.30 & 0.04 \\
Specimen No. 20 & 8 years & 0.08 & 0.005 \\
\hline
\end{tabular}

By 3 months a cross section of the ligamentum teres shows the typical zonal arrangement (Fig. 4) recognizable up to the sixth or seventh decade of life, which is as follows:

Zone 1: A tiny central residual lumen of the intraabdominal umbilical vein; occasionally doubled.

Zone 2: A zone of fine connective tissue formed by sub-intimal proliferation.

Zone 3: The elastic fibres of the original intima closely packed into an irregular ring. In later life a wide zone of longitudinal smooth muscle appears in the periphery of this ring.

Zone 4: The outer fibro-elastic zone, formed from the fibro-muscular media of the vein, and forming the bulk of the ligamentum teres. It contains, from 20 years onwards, a few longitudinal smooth muscle fibres and fat is laid down in its periphery.

The above sequence of changes is typical of sections taken at about the middle of the ligamentum teres or umbilical vein. At the hepatic extremity of the ligament the changes inside the elastic ring are modified by the absence or scant formation of new fibrous tissue. The residual lumen is therefore larger and is bounded by the musculo-elastic ring. At the umbilical extremity, however, the formation of the central core of fibrous tissue is more marked and all the residual lumen is completely obliterated. Furthermore, the fibro-elastic zone of the ligamentum teres forms a series of irregular tails 
(Robin, 1860) which are connected to similar tails formed from the urachus and the obliterated intraabdominal umbilical arteries. The material used in

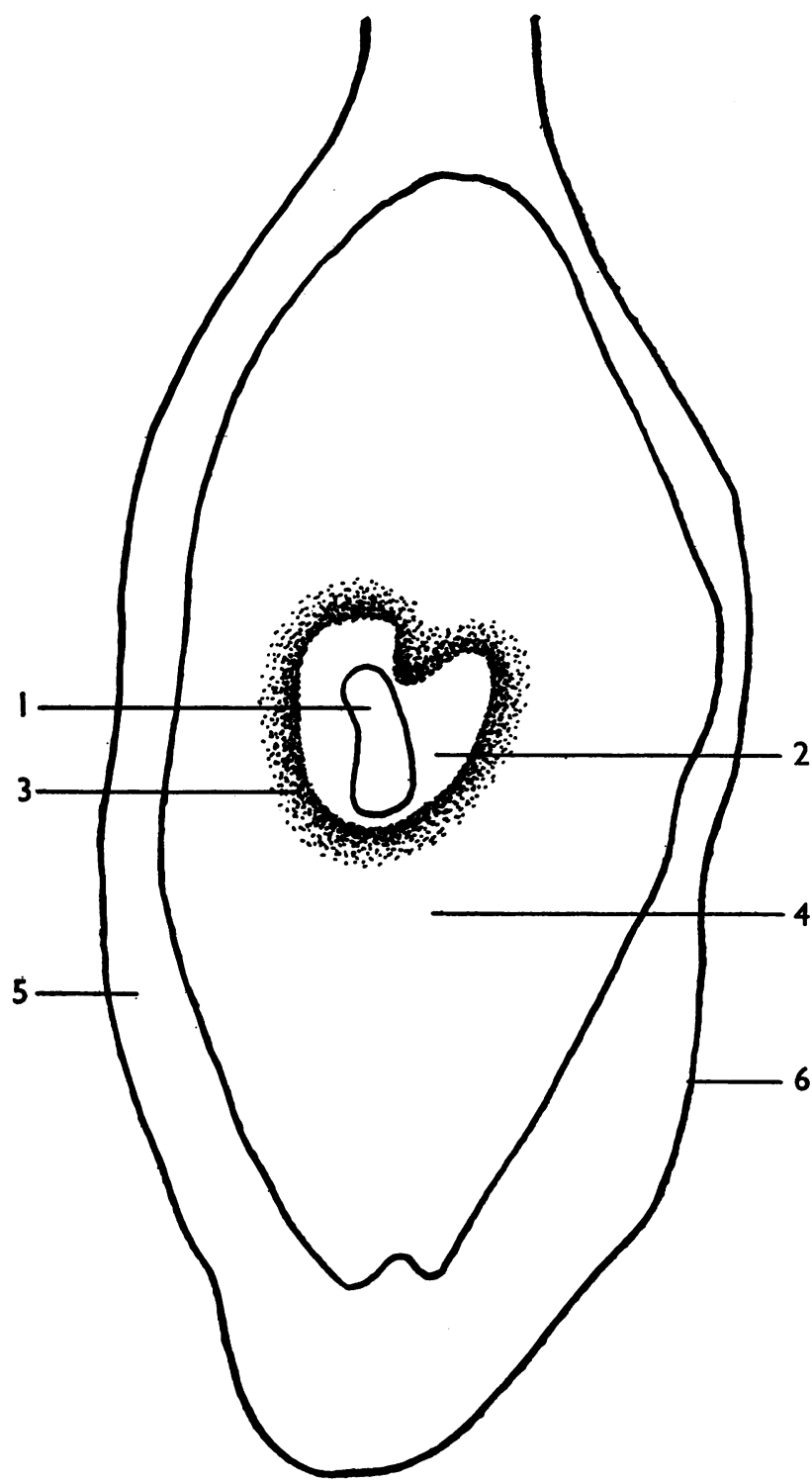

FIG. 4.-Tracing of a section of the ligamentum teres, aged 3 months, showing the typical zonal arrangement. (See Fig. 3, 4.) $\times 25$.

1. Central residual lumen.

2. Connective tissue zone.

3. Musculo-elastic zone.

4. Fibro-elastic zone.

5. Extra-peritoneal tissue.

6. Peritoneum of the falciform ligament.

this present study did not permit investigation of the formation of these tails, which would appear, however, to be related to the growth in length of the ligamentum teres.

\section{DISCUSSION}

The dimensional and histological changes characterizing the intra-abdominal umbilical vein during the first 48 hours of life indicate that this vein has already partially contracted. Because of the scanty amount of smooth muscle in its wall the contraction is less complete than that which occurs in the umbilical arteries (Mollison et al., 1948) or in the ductus arteriosus (Kennedy and Clark, 1941, Figs. 5 and 6). In these arterial vessels the lumen is almost completely obliterated so that the blood stops flowing. It is noteworthy that the intra-abdominal umbilical vein receives tributaries (the veins of Burow and its own vena venarum) which empty a small amount of blood into the vein after the cutting off of the placental inflow.

The average diameter of the lumen of the partially contracted vein, measured after fixation and sectioning, is $1.1 \mathrm{~mm}$. This is in keeping with the ability to pass a polythene catheter of $1.0 \mathrm{~mm}$. internal bore for the purpose of exchange transfusion. Further, a lumen of this order of size is found up to the thirteenth day after birth, so that the limiting factor in the utilization of this route for exchange transfusion is the formation of the umbilical scar and the falling off of the stump of the umbilical cord. The vein has, indeed, been utilized as late as the sixth day after birth (Diamond, 1954).

Anatomical obliteration of the lumen is well marked by the twenty-eighth day after birth but begins at, or just before, birth. Two main processes are apparent in such obliteration, viz., (a) a subintimal proliferation which fills the periphery of the lumen with new fibrous tissue, (b) the formation and subsequent contraction of coarse collagen fibres in the fibro-muscular media, such tissue forming the bulk of the completed ligamentum teres. These processes are further assisted by a side-to-side flattening of the partially contracted vein. There is no evidence to suggest that thrombosis and subsequent clot organization ever play any part in the normal process of obliteration. A marked gradient occurs in the intensity of obliterative change along the length of the vein, being maximal at the umbilical, and minimal towards the hepatic, end. This gradient appears to be related to the site of entry of the veins of Burow, which are so valved as to direct blood from the abdominal parietes towards the portal venous system. It is possible that this vascular connexion affords a route for the 
haemal spread of cord sepsis into the liver of the newborn.

The evidence obtained from present material supports the view of Baumgarten (1891) that the lumen of the intra-abdominal umbilical vein is normally retained in the hepatic end of the ligamentum teres. It affords no support to the view of Wertheimer (1886) that the central lumen is caused by revascularization of the central fibroustissue core. The double or treble lumina which he regarded as diagnostic of new capillary formation are readily explicable by the irregular mode of growth of the tissue formed by sub-intimal proliferation.

\section{SUMMARY}

Soon after birth the intra-abdominal umbilical vein undergoes partial contraction and its lumen is reduced by about one-half.

Subsequent anatomical obliteration is incomplete and a tiny residuum of the original lumen persists at the hepatic end of the ligamentum teres.

Anatomical obliteration is a combination of (a) sub-intimal proliferation, (b) the formation and subsequent shrinkage of coarse collagen fibres in the fibro-muscular media, and (c) side-to-side flattening of the vein.
Thrombosis and subsequent clot-organization play no part in the obliterative process under normal conditions.

The elastic fibres of the venous intima persist, in the centre of the ligamentum teres, up to the sixth and seventh decades.

My thanks are due to Professor A. J. E. Cave for constant advice and encouragement. Particular gratitude is expressed to Dr. A. M. Barrett, Department of Pathology, University of Cambridge, and to his staff, for very generous and consistent supplies of material. To the departmental technicians, Miss J. Stedman and Mr. A. E. Westwood, are due respectively the sections and the photomicrographs.

\section{REFERENCES}

Barclay, A. E., Franklin, K. J. and Prichard, M. M. L. (1944). The Foetal Circulation and Cardiovascular System, and the Changes that they Undergo at Birth, pp. 74-75, 160-161, 219-220. Oxford.

Oxford.
Baumgarten, (1891). Arb. path. Anat. Inst., Tubingen, $1,1$. Butler, H. (195i). J. Anat., Lond., 85, 430.

Butler, (1952). Thorax, 7, 159 .

Diamond, L. K. (1947).' Proc. roy. Soc. Med., 40, 546.

- (1954). Personal communication.

Kennedy, J. A. and Clark, S. L. (1941). Anat. Rec., 79, 349.

Mennedy, J. A. and Clark, S. L. (1941). Anat. Rec., 79, 349. Coun. Memo. No. 19, pp. 43-45.

Robin, C. (1860). Mém. Acad. Méd., Paris, 24, 391.

Scammon, R. E. and Norris, E. H. (1918). Anat. Rec., 15, 165

Segall, H. N. (1923). Surg. Gynec. Obstet., 37, 152.

Sidbury, J. B. (1923) Amer. J. Dis, Child, 25, 290.

Wertheimer, E. (1886). J. Anat., Paris, 22, 1. 